\title{
Caracterização do usuário de substâncias psicoativas e a importância do serviço de álcool e outras drogas
}

\author{
Characterization of the user of psychoactive substances and the importance of the alcohol \\ service and other drugs
}

Caracterización del usuario de sustancias psicoactivas y la importancia del servicio de alcohol y otras drogas

Paulo Henrique Santana Feitosa Sousa ${ }^{*}$, Thaynara Fontes Almeida ${ }^{2}$, Marcel Vinicius Cunha Azevedo ${ }^{1}$, Ruth Cristini Torres ${ }^{1}$, Bárbara da Silva Nunes ${ }^{1}$, Maria Aparecida Bispo Novais dos Santos e Santos ${ }^{1}$, Douglas Vinícius dos Santos Feitosa ${ }^{1}$, Gilverton Melo Santos Júnior ${ }^{1}$.

\section{RESUMO}

Objetivo: Pesquisar nas evidências científicas o perfil do usuário de substâncias psicoativas, bem como, esclarecer a importância do serviço de álcool e outras drogas. Métodos: Trata-se de uma revisão integrativa da literatura de caráter descritivo usando publicações das seguintes bases de dados: Literatura Latino-Americana do Caribe em Ciências da Saúde (LILACS), Scientific Electronic Library Online (SciELO) e Sistema Online de Busca e Análise de Literatura (MEDLINE). Resultados: A amostra foi constituída por 16 artigos, sendo 6 estudos de caráter transversal (38\%), 4 sendo quantitativos (25\%), sendo 3 artigos descritivos (19\%), 2 fenomenológicos (12\%) e 1 epidemiológico (6\%). Conclusão: Constatou-se que a maior parte desse público eram homens solteiros com apenas um filho, com a faixa etária entre 20 e 60 anos, a maioria não havia concluído o ensino fundamental, apresentando entre 1 e 2 salários mínimos, predominando a cor parda e o álcool como a droga lícita mais utilizada. Nesse contexto, os Centros de Atenção Psicossocial tornam-se extremamente importantes pois visam garantir aos usuários uma nova forma de cuidados, possibilitando uma nova perspectiva da reinserção social, eliminando o foco da atenção centrada no controle e na repressão, por meio das atividades como participação em grupos ou oficinas e outras atividades educativas.

Palavras-chave: Usuário de drogas, Transtornos relacionados ao uso de substâncias, Centros de tratamento de abuso de substâncias.

\section{ABSTRACT}

Objective: The present study aims to search the scientific evidence for the profile of the user of psychoactive substances, as well as to clarify the importance of the alcohol and other drugs service. Methods: It is an integrative literature review of a descriptive nature using publications from the following databases: Latin American Caribbean Literature in Health Sciences (LILACS), Scientific Electronic Library Online (SciELO) and Online Literature Search and Analysis System (MEDLINE). Results: He sample consisted of 16 articles, with 6 cross-sectional studies (38\%), 4 being quantitative (25\%), 3 descriptive articles (19\%), 2 phenomenological (12\%) and 1 epidemiological (6\%). Conclusion: It was found that most of this audience were single men with only one child, aged between 20 and 60 years, most had not completed elementary school, presenting between 1 and 2 minimum wages, with predominance of brown and alcohol as the most used legal drug. In this context, psychosocial care center become extremely important because they aim to guarantee users a new form of health care, enabling a new perspective of social reintegration, eliminating the focus of attention centered on control and repression, through activities such as participation in groups or workshops and other educational activities.

Keywords: Drug user, Disorders related to substance use, Substance abuse treatment centers.

\section{RESUMEN}

Objetivo: El presente estudio tiene como objetivo utilizar sustancias químicas o el perfil de usuario de sustancias psicoactivas, así como aclarar la importancia del servicio de alcohol y otras drogas. Métodos: Esta es una revisión de literatura integradora de naturaleza descriptiva que utiliza publicaciones de las siguientes bases de datos: Literatura Latinoamericana del Caribe en Ciencias de la Salud (LILACS), Biblioteca Electrónica Científica en Línea (SciELO) y Sistema de Búsqueda y Análisis en Línea de literatura (MEDLINE). Resultados: 16 artículos desarrollaron una muestra, con 6 estudios transversales (38\%), 4 cuantitativos (25\%), 3 descriptivos (19\%), 2 fenomenológicos (12\%) y 1 epidemiológicos (6\%). Conclusión: Se encontró que la mayoría de esta audiencia eran hombres solteros con un solo hijo, de entre 20 y 60 años, la mayoría no había completado la educación primaria, presentando entre 1 y 2 salarios mínimos, con predominio de los morenos y el alcohol como la droga legal más consumida. En este contexto, los Centros de Atención Psicosocial cobran suma importancia porque apuntan a garantizar a los usuarios una nueva forma de cuidado, posibilitando una nueva perspectiva de reinserción social, eliminando el foco de atención centrado en el control y la represión, a través de actividades como la participación. en grupos o talleres y otras actividades educativas.

Palabras clave: Usuário de drogas, Transtornos relacionados ao uso de substâncias, Centros de tratamento de abuso de substâncias.

${ }^{1}$ Centro Universitário Estácio de Sergipe, Aracaju - SE.*E-mail: paulo.henriique@hotmail.com

2Universidade Federal de Sergipe (UFS), Aracaju - SE.

SUBMETIDO EM: 7/2020

ACEITO EM: $8 / 2020$

PUBLICADO EM: 12/2020 


\section{INTRODUÇÃO}

O consumo de drogas é uma preocupação mundial, devido a sua alta incidência e riscos à saúde, provenientes do seu uso. Mundialmente, o uso de drogas caracteriza-se como um grave problema social e de saúde pública, causando danos consideráveis ao indivíduo, além de desafios no sistema de controle da violência advinda do tráfico e da rápida evolução de novas substâncias psicoativas (ANDRETTA I, et al., 2018).

Segundo Chaves JJF e Khenti A (2019), cerca de 5\% da população adulta mundial relataram usar drogas pelo menos uma vez na vida. Ao longo dos últimos 3 anos podemos observar que 243 milhões de pessoas consumiram substâncias ilícitas, possuindo a faixa etária entre 15 e 64 anos de idade. Desta população, uma em cada 200 pessoas adultas de todo o mundo, é usuária regular de drogas (LUCCHESE R, et al., 2017). As drogas psicoativas são definidas como aquelas que atuam no sistema nervoso central, podendo causar alterações comportamentais, cognitivas e no humor do usuário. Com isso, temos uma diversidade de drogas que podem causar efeitos sobre a saúde do indivíduo, podendo ser classificadas como depressoras, estimulantes, perturbadoras do sistema nervoso central (NUNES JT, et al., 2017).

No Brasil, o álcool se apresenta como a substância psicoativa mais utilizada, ou pelo menos a mais experimentada. Devido ao seu caráter lícito, o que favorece seu consumo em excesso. Quando se fala em jovens, o álcool e o tabaco são as primeiras experimentadas, em geral muito precocemente e sem limite de doses. O usuário que se torna dependente do álcool passa a buscar efeitos cada vez mais intensos nas substâncias ilícitas, tornando-se assim um poliusuário (WEISS CV, et al., 2018).

O uso contínuo de substâncias psicoativas gera alterações significativas na vida do usuário, sendo considerado como consequência de um transtorno da pessoa por inteiro, que levam a mudanças em diversas áreas da sua vida, no meio familiar, psicossocial, comportamental, afastamento do mercado de trabalho, envolvimentos em atos violentos, problemas no funcionamento do cérebro e comorbidades (FERNANDES MA, et al., 2018).

A Reforma Psiquiátrica Brasileira foi um movimento social, político e jurídico que extinguiu a proposta de internação manicomial como tratamento central para as pessoas com transtornos mentais. Este movimento foi reafirmado com a Lei 10.216/01, que prevê novas estratégias e alternativas de atenção à saúde mental, bem como, maiores investimentos na área, sendo de modo articulado e integrado com base no território, como no caso da Rede de Atenção Psicossocial, que diz respeito à Portaria № 3.088 (LIMA DWC, et al., 2018).

O Ministério da Saúde tem buscado, em conjunto com políticas sociais, executar e aprimorar a qualidade da atenção a esses usuários por meio de serviços que substituam o modelo manicomial. Os Serviços de Redução de Danos (RD) e os Centros de Atenção Psicossocial Álcool e Drogas (CAPS AD), objetivam um cuidado de forma integral, articulado e em todos os níveis de atenção em saúde, fortalecendo o cuidado extra hospitalar (WEISS CV, et al., 2018).

Os CAPS são serviços destinados a articular as ações de saúde mental em rede e junto à atenção básica, ambulatórios, leitos de internação em hospitais gerais e ações de suporte e reabilitação psicossocial. Eles perpassam a lógica da hierarquização e se organizam através dos diferentes níveis de atenção à saúde. Prestam atendimento especializado dos casos de transtornos mentais, são responsáveis pelo acompanhamento dos pacientes nas unidades de internação nos hospitais gerais, atuam em nível de atenção primária, no acompanhamento e apoio matricial de casos nas Unidades Básicas de Saúde (UBS), percorrendo, portanto, todos os níveis de complexidade do Sistema Único de Saúde (QUINDERÁ PH, et al., 2014).

A justificativa para a realização deste estudo surgiu a partir da prática de estágio em saúde mental vivenciado pelos pesquisadores, em que se observou um grande número de usuários de álcool e outras drogas. Acredita-se que o estudo contribua na compreensão da importância do serviço de álcool e drogas e da identificação do perfil dos pacientes. Assim, o presente estudo tem como objetivo pesquisar nas evidências científicas o perfil do usuário de substâncias psicoativas, bem como, esclarecer a importância do serviço de álcool e outras drogas. 


\section{MÉTODOS}

O estudo utiliza como método a revisão integrativa da literatura de caráter descritivo. Para guiar a pesquisa, formularam-se as seguintes questões norteadoras: quem são os usuários de substâncias psicoativas? Qual a importância do serviço de álcool e outras drogas na promoção de saúde?

A pesquisa das publicações foi realizada de junho de 2019 a junho de 2020, busca as publicações indexadas nas seguintes bases de dados: Literatura Latino-Americana do Caribe em Ciências da Saúde (LILACS), Scientific Electronic Library Online (SciELO) e Sistema Online de Busca e Análise de Literatura (MEDLINE). A busca científica foi realizada através da utilização dos descritores em saúde (DeCS): usuário de drogas; transtornos relacionados ao uso de substâncias; centros de tratamento de abuso de substâncias, combinados por meio do operador boleano AND.

O processo de identificação, seleção e inclusão dos estudos primários se deu em três etapas. Na primeira foi realizada a retirada de 12 artigos duplicados de um total de 214. Em seguida, procedeu-se à leitura dos títulos e resumos dos 202 artigos, à luz dos critérios de inclusão. Destes, foram retirados 136 artigos por não responderem as questões norteadoras desta revisão, sendo selecionados 65 artigos para a leitura na íntegra configurando a terceira etapa, resultando uma amostra final foi de 17 artigos (Figura 1).

Figura 1 - Fluxograma de identificação, seleção e inclusão dos estudos da revisão integrativa.

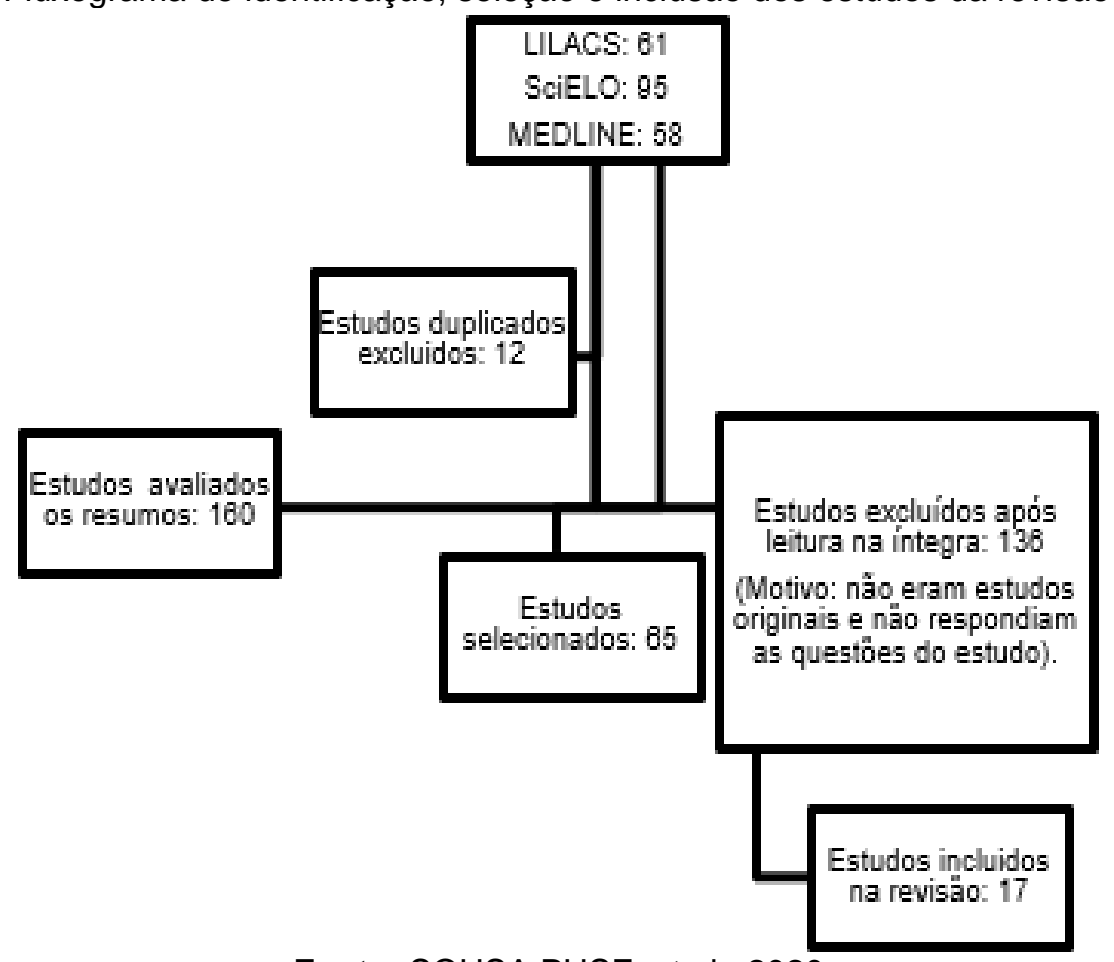

Fonte: SOUSA PHSF, et al., 2020.

Os critérios de inclusão foram: artigos originais publicados na íntegra, disponíveis eletronicamente, em língua portuguesa e inglesa, publicados entre os anos de 2015 a 2020. Sendo excluídas: teses, dissertações e monografias.

O estudo foi desenvolvido respeitando a lei dos direitos autorais, lei 12.853/13, que dispõe em seu Art. 1ํ. Essa Lei regula os direitos autorais, entendendo-se sob esta denominação os direitos de autor e os que lhes são conexos.

\section{RESULTADOS}

Do material obtido, foi elaborado um instrumento para coleta de informações, a fim de responder às questões norteadoras desta revisão, em ordem decrescente, composto pelos seguintes itens: título do artigo, objetivo, metodologia, autor e ano de publicação (Quadro 1). 
Revista Eletrônica Acervo Saúde / Electronic Journal Collection Health | ISSN 2178-2091

Quadro 1 - Artigos utilizados para o embasamento teórico desta pesquisa.

\begin{tabular}{|c|c|c|c|c|}
\hline № & Autor(es)/Data & Título & Objetivo & Metodologia \\
\hline 1 & $\begin{array}{l}\text { Bakari I e Mann R } \\
\qquad(2019)\end{array}$ & $\begin{array}{c}\text { Atitudes em relação às pessoas que } \\
\text { abusam de drogas em uma comunidade } \\
\text { urbana de woodbrook no Porto de Espanha } \\
\text {-trinidad \& tobago. }\end{array}$ & $\begin{array}{c}\text { Este estudo apresentará insights sobre a situação na } \\
\text { amostra pesquisada, no que se refere às atitudes em } \\
\text { relação às pessoas que abusam de álcool por idade e } \\
\text { sexo e constituiria uma plataforma sobre a qual, novas } \\
\text { pesquisas podem ser conduzidas por pesquisadores } \\
\text { da área de drogas. }\end{array}$ & Estudo transversal. \\
\hline 2 & $\begin{array}{l}\text { Chaves JJF e Khenti } \\
\text { A (2019) }\end{array}$ & $\begin{array}{l}\text { Conhecimento das consequências e uso de } \\
\text { drogas para estudantes universitários da } \\
\text { Costa Rica. }\end{array}$ & $\begin{array}{c}\text { Determinar a relação entre o conhecimento das } \\
\text { consequências e o uso de drogas em estudantes de } \\
\text { graduação de uma universidade em San José, Costa } \\
\text { Rica. }\end{array}$ & Estudo transversal. \\
\hline 3 & $\begin{array}{l}\text { Fernandes MA, et al. } \\
\qquad(2018)\end{array}$ & $\begin{array}{l}\text { Caracterização de dependentes químicos } \\
\text { em tratamento em uma comunidade } \\
\text { terapêutica. }\end{array}$ & $\begin{array}{c}\text { Caracterizar o perfil de indivíduos com dependência } \\
\text { química de uma Comunidade Terapêutica. }\end{array}$ & $\begin{array}{l}\text { Estudo quantitativo, } \\
\text { descritivo e } \\
\text { exploratório. }\end{array}$ \\
\hline 4 & $\begin{array}{l}\text { Lima DWC, et al. } \\
\qquad(2018) .\end{array}$ & $\begin{array}{l}\text { Práticas dos enfermeiros às pessoas em } \\
\text { crise psíquica em decorrência do uso } \\
\text { abusivo de drogas. }\end{array}$ & $\begin{array}{l}\text { Compreender as práticas dos enfermeiros em serviço } \\
\text { de urgência e emergência às pessoas em situação de } \\
\text { crise psíquica decorrente do uso abusivo de drogas. }\end{array}$ & $\begin{array}{l}\text { Pesquisa descritiva e } \\
\text { abordagem } \\
\text { qualitativa. }\end{array}$ \\
\hline 5 & $\begin{array}{l}\text { Siqueira DF, et al. } \\
\qquad(2018)\end{array}$ & $\begin{array}{l}\text { Motivos atribuídos por usuários à procura } \\
\text { de tratamento em um centro de atenção } \\
\text { psicossocial álcool e drogas. }\end{array}$ & $\begin{array}{l}\text { Compreender os motivos atribuídos por usuários de } \\
\text { substâncias psicoativas à procura de tratamento em } \\
\text { um Centro de Atenção Psicossocial Álcool e Drogas. }\end{array}$ & $\begin{array}{l}\text { Estudo } \\
\text { fenomenológico à luz } \\
\text { da fenomenologia } \\
\text { social. }\end{array}$ \\
\hline 6 & $\begin{array}{l}\text { Weiss CV, et al. } \\
\qquad(2018)\end{array}$ & $\begin{array}{l}\text { Prevalência da dependência de álcool em } \\
\text { usuários de substâncias psicoativas. }\end{array}$ & $\begin{array}{l}\text { Analisar a prevalência da dependência de álcool em } \\
\text { usuários de substâncias psicoativas do município de } \\
\text { Pelotas-Rio Grande do Sul. }\end{array}$ & $\begin{array}{l}\text { Pesquisa transversal, } \\
\text { exploratória de } \\
\text { abordagem } \\
\text { quantitativa. }\end{array}$ \\
\hline 7 & $\begin{array}{l}\text { Andretta I, et al. } \\
\qquad(2018)\end{array}$ & $\begin{array}{l}\text { Sintomas de depressão, ansiedade e } \\
\text { estresse em usuários de drogas em } \\
\text { tratamento em comunidades terapêuticas. }\end{array}$ & $\begin{array}{c}\text { Avaliar os níveis de depressão, ansiedade e estresse } \\
\text { em homens e mulheres em tratamento em } \\
\text { Comunidades Terapêuticas, relacionando tais níveis } \\
\text { com o tipo de substância utilizada e dados } \\
\text { sociodemográficos. }\end{array}$ & $\begin{array}{l}\text { Estudo quantitativo, } \\
\text { avaliativo, } \\
\text { transversal, descritivo } \\
\text { e exploratório }\end{array}$ \\
\hline 8 & $\begin{array}{l}\text { Tassinari TT, et al. } \\
\qquad(2018)\end{array}$ & $\begin{array}{l}\text { Caracterização de mulheres em tratamento } \\
\text { devido ao uso de drogas. }\end{array}$ & $\begin{array}{l}\text { Caracterizar a população de mulheres que realizaram } \\
\text { tratamento em um Centro de Atenção Psicossocial } \\
\text { Álcool e Outras Drogas. }\end{array}$ & $\begin{array}{l}\text { Estudo quantitativo, } \\
\text { documental, } \\
\text { retrospectivo. }\end{array}$ \\
\hline
\end{tabular}

REAS/EJCH | Vol.12(12) | e4846 | DOI: https://doi.org/10.25248/reas.e4846.2020 Página 4 de 11 
Revista Eletrônica Acervo Saúde / Electronic Journal Collection Health | ISSN 2178-2091

\begin{tabular}{|c|c|c|c|c|}
\hline № & Autor(es)/Data & Título & Objetivo & Metodologia \\
\hline 9 & $\begin{array}{l}\text { Boska GA, et al. } \\
\qquad(2018)\end{array}$ & $\begin{array}{c}\text { Mudanças percebidas por usuários de } \\
\text { centros de atenção psicossocial em álcool e } \\
\text { outras drogas. }\end{array}$ & $\begin{array}{l}\text { Avaliar se os usuários dos Centros de Atenção } \\
\text { Psicossocial em Álcool e Outras Drogas percebem } \\
\text { mudanças com o tratamento recebido e quais as } \\
\text { variáveis associadas a tais mudanças. }\end{array}$ & $\begin{array}{l}\text { Estudo quantitativo, } \\
\text { avaliativo, } \\
\text { transversal, descritivo } \\
\text { e exploratório }\end{array}$ \\
\hline 10 & $\begin{array}{l}\text { Carvalho M R S, et al. } \\
\qquad(2017)\end{array}$ & $\begin{array}{l}\text { Motivações e repercussões do consumo de } \\
\text { crack: o discurso coletivo de usuários de um } \\
\text { centro de atenção psicossocial. }\end{array}$ & $\begin{array}{c}\text { Identificar as motivações e repercussões relacionadas } \\
\text { ao consumo do crack por pessoas atendidas em um } \\
\text { Centro de Atenção Psicossocial Álcool e Drogas } \\
\text { (CAPS AD) no interior da Bahia. }\end{array}$ & $\begin{array}{l}\text { Estudo descritivo, de } \\
\text { abordagem } \\
\text { qualitativa. }\end{array}$ \\
\hline 11 & $\begin{array}{l}\text { Nunes JT, et al. } \\
\qquad(2017)\end{array}$ & $\begin{array}{l}\text { Assistência de enfermagem aos usuários de } \\
\text { drogas psicoativas em sofrimento psíquico: } \\
\text { relato de experiência. }\end{array}$ & $\begin{array}{l}\text { Relatar a experiência de acadêmicas de enfermagem } \\
\text { quanto à assistência oferecida a dependentes } \\
\text { químicos em sofrimento psíquico e discutir os } \\
\text { prejuízos psicossociais experimentados por eles. }\end{array}$ & $\begin{array}{l}\text { Estudo descritivo de } \\
\text { abordagem } \\
\text { qualitativa, do tipo } \\
\text { relato de experiência. }\end{array}$ \\
\hline 12 & $\begin{array}{l}\text { Tavares MLO, et al. } \\
\qquad(2017)\end{array}$ & $\begin{array}{l}\text { Perfil de adolescentes e vulnerabilidade } \\
\text { para o uso de álcool e outras drogas. }\end{array}$ & $\begin{array}{l}\text { Caracterizar o perfil de adolescentes e vulnerabilidade } \\
\text { para o uso de álcool e outras drogas. }\end{array}$ & $\begin{array}{l}\text { Estudo descritivo, } \\
\text { exploratório, } \\
\text { transversal. }\end{array}$ \\
\hline 13 & $\begin{array}{l}\text { Cosentino SF, et al. } \\
\qquad(2017)\end{array}$ & $\begin{array}{l}\text { Características de cuidadores familiares e } \\
\text { de usuários de drogas. }\end{array}$ & $\begin{array}{c}\text { Investigar as características sociodemográficas, } \\
\text { econômicas, de saúde, atividade de cuidado e apoio } \\
\text { social dos cuidadores familiares e de dependentes de } \\
\text { substância psicoativa ilícita. }\end{array}$ & $\begin{array}{l}\text { Estudo } \\
\text { epidemiológico, com } \\
\text { desenho transversal. }\end{array}$ \\
\hline 14 & $\begin{array}{l}\text { Reis LM e Oliveira } \\
\text { MLF (2017) }\end{array}$ & $\begin{array}{c}\text { Vulnerabilidade social em famílias que } \\
\text { convivem com comportamento aditivo por } \\
\text { tempo prolongado. }\end{array}$ & $\begin{array}{l}\text { Analisar a vulnerabilidade social de famílias que } \\
\text { convivem por tempo prolongado com o } \\
\text { comportamento aditivo de um de seus membros. }\end{array}$ & Estudo transversal. \\
\hline 15 & $\begin{array}{l}\text { Lucchese R, et al. } \\
\qquad(2017)\end{array}$ & $\begin{array}{c}\text { Transtorno mental comum entre indivíduos } \\
\text { que abusam de álcool e drogas: estudo } \\
\text { transversal. }\end{array}$ & $\begin{array}{l}\text { Estimar a prevalência da probabilidade de transtorno } \\
\text { mental comum entre indivíduos que abusam de álcool } \\
\text { e outras drogas. }\end{array}$ & Estudo transversal. \\
\hline 16 & $\begin{array}{l}\text { Sena ELS, et al. } \\
\qquad(2017) .\end{array}$ & $\begin{array}{l}\text { Ambiguidade do cuidado na vivência do } \\
\text { consumidor de drogas. }\end{array}$ & $\begin{array}{l}\text { Compreender a percepção de usuários de um Centro } \\
\text { de Atenção Psicossocial sobre o cuidado no contexto } \\
\text { do consumo de drogas. }\end{array}$ & $\begin{array}{l}\text { Estudo fundamentado } \\
\text { na Fenomenologia. }\end{array}$ \\
\hline 17 & $\begin{array}{l}\text { Abreu AMM, et al. } \\
\qquad(2016)\end{array}$ & $\begin{array}{c}\text { Perfil do consumo de substâncias } \\
\text { psicoativas e sua relação com as } \\
\text { características sociodemográficas: uma } \\
\text { contribuição para intervenção breve na } \\
\text { atenção primária à saúde, Rio de Janeiro, } \\
\text { Brasil. }\end{array}$ & $\begin{array}{l}\text { Analisar o perfil de consumo de substâncias } \\
\text { psicoativas nos últimos três meses e sua associação } \\
\text { com as variáveis sociodemográficas. }\end{array}$ & Estudo transversal \\
\hline
\end{tabular}

Fonte: SOUSA PHSF, et al., 2020.

REAS/EJCH | Vol.12(12) | e4846 | DOI: https://doi.org/10.25248/reas.e4846.2020 Página $\mathbf{5}$ de 11 
Constatou-se a predominância de 6 estudos de caráter transversal (35\%), 4 sendo quantitativos (24\%), sendo 4 artigos descritivos (24\%), 2 fenomenológicos (12\%), 1 epidemiológico (6\%) (Figura 2).

Figura 2 - Gráfico de classificação dos artigos em relação ao tipo de metodologia adotada.

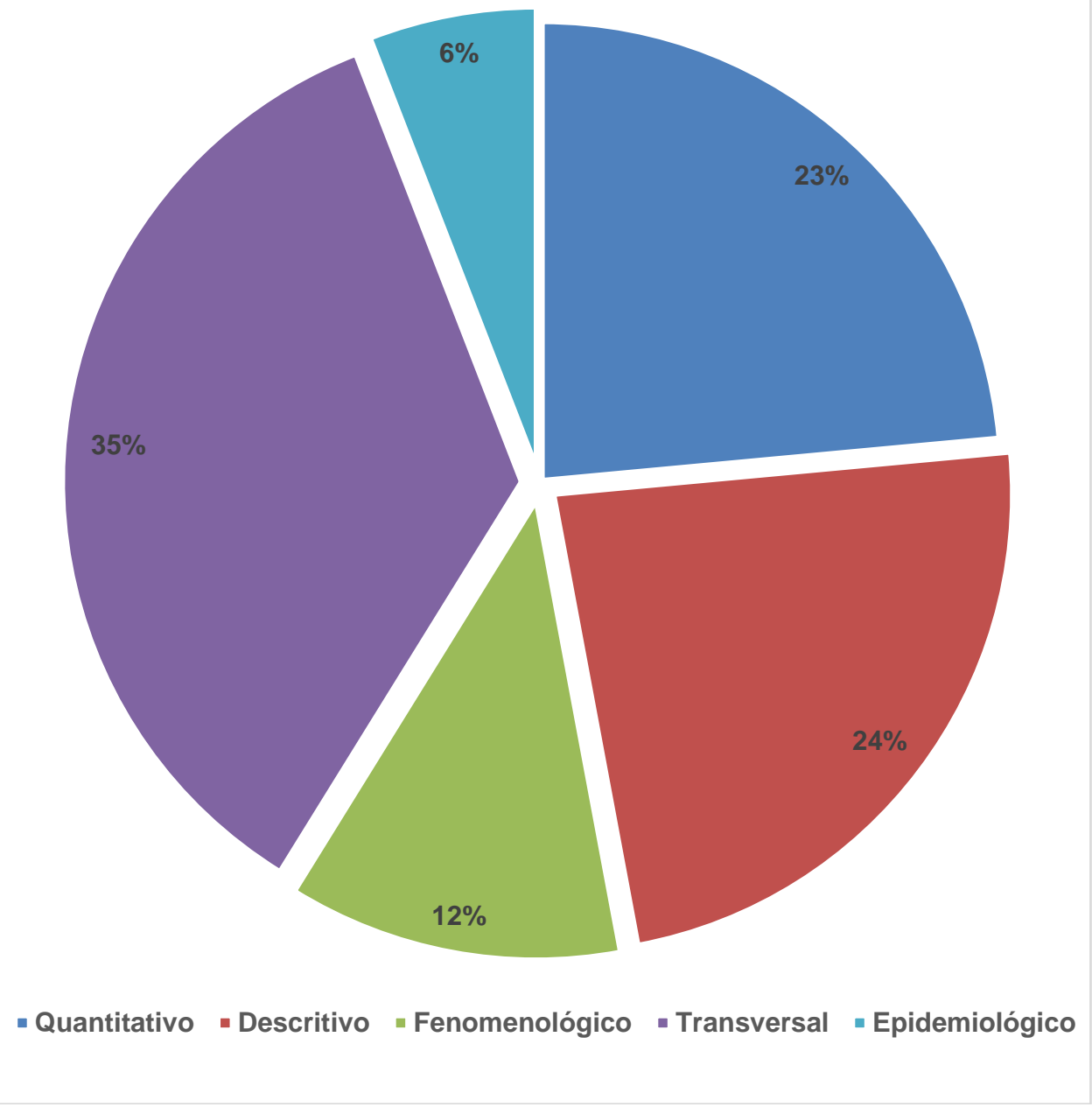

Fonte: SOUSA PHSF, et al., 2020.

Quando se trata de consumo de substâncias psicoativas, a prevalência é maior entre o sexo masculino, havendo uma variação na média de idade de 19 a 49 anos (Tabela 1).

Tabela 1 - Dados numéricos por gênero e idade dos usuários de drogas.

\begin{tabular}{cccc}
\hline Autor/Ano & Gênero & Porcentagem (\%) & Idade Média \\
\hline \multirow{2}{*}{ Bakari I e Mann R (2019). } & Feminino & $38,3 \%$ & \multirow{2}{*}{19 a 29 anos } \\
& Masculino & $35,3 \%$ & \multirow{2}{*}{30 a 49 anos } \\
\hline \multirow{2}{*}{ Weiss CV, et al. (2018). } & Feminino & $16,2 \%$ & \multirow{2}{*}{43,9 anos } \\
\hline \multirow{2}{*}{ Boska GA, et al. (2018). } & Masculino & $83,8 \%$ & $20,9 \%$ \\
& Feminino & $79,1 \%$ & \\
\hline
\end{tabular}

Fonte: SOUSA PHSF, et al., 2020.

Ao analisar o grau de escolaridade dos usuários de drogas, a prevalência foi daqueles que possuem o ensino fundamental incompleto, sendo assim observado que quanto maior a escolaridade, menor o consumo dessas substâncias (Tabela 2). 
Tabela 2 - Dados relacionados ao nível de escolaridade dos usuários de drogas.

\begin{tabular}{ccc}
\hline Autor/ Ano & Nível de Escolaridade & $\%$ \\
\hline \multirow{2}{*}{ Abreu AMM, et al., 2018 } & Fundamental Incompleto & 34 \\
& Fundamental Completo & 16,4 \\
& Médio Completo & 22,6 \\
& Médio Incompleto & 15,9 \\
& Superior Completo & 2.7 \\
\hline \multirow{3}{*}{ Fernandes MA, et al., 2018 } & Fundamental Incompleto & 45,4 \\
& Fundamental Completo & 9,1 \\
& Médio Incompleto & 18,1 \\
Boska GA, et al., 2018 & Médio Completo & 22,8 \\
& Superior Incompleto & 4,6 \\
& Superior Completo & 0,0 \\
\hline \multirow{3}{*}{ Cosentino SF, et al., 2017 } & Fundamental Incompleto & 29,7 \\
& Médio incompleto & 25,5 \\
& Técnico Completo & 25,5 \\
& Superior Incompleto & 19,4 \\
& Superior Completo & 19,4 \\
\hline
\end{tabular}

Fonte: SOUSA, PHSF, et al., 2020.

Dentre as motivações iniciais para o consumo de substâncias psicoativas citadas acima, encontram-se em maior prevalência a curiosidade, fatores estressantes e influências dos amigos e da família (Tabela 3).

Tabela 3 - Dados pela motivação inicial do consumo de drogas.

\begin{tabular}{cc}
\hline Autor/ Ano & Motivação \\
\hline Andretta I, et al. (2018) & Eventos Estressores \\
\hline Tassinari TT, et al. (2018) & $\begin{array}{c}\text { Influência dos usuários } \\
\text { Círculo de amigos } \\
\text { Influência da família } \\
\text { Meio social }\end{array}$ \\
\hline Sena ELS, et al. (2017) & Curiosidade \\
& Excesso de trabalho \\
& Efeitos produzidos pelas drogas \\
\hline Nunes JT, et al. (2017) & Hedonismo \\
& Curiosidade \\
& Alívio de dor \\
& Sofrimento \\
\hline
\end{tabular}

Fonte: SOUSA PHSF, et al., 2020. 


\section{DISCUSSÃO}

\section{Perfil dos usuários de substâncias psicoativas}

A Organização Mundial de Saúde (OMS) em parceria com a United Nations Office on Drugs and Crime (UNODC), em seu relatório sobre a atenção e o tratamento às drogas, revelou que 205 milhões de pessoas consomem drogas ilícitas no mundo, das quais 25 milhões se caracterizam no quadro de dependência, indicando assim, o consumo das drogas como grupo dos 20 principais fatores de risco para a saúde no mundo e um dos 10 principais fatores nos países em desenvolvimento (ABREU AMM, et al., 2016). Na contemporaneidade, o uso de forma abusiva dessas substâncias deixou de ser um costume ou um vício, e configura-se como uma enfermidade crônica, progressiva e fatal, prejudicando bem-estar físico, mental, emocional e social do usuário (BERALDO JB, et al., 2018).

Em relação aos dados referentes ao sexo, a predominância é da população masculina sendo $(33,4 \%)$ dos usuários, em contraposição, $78,1 \%$ dessa população é composta por mulheres. Um estudo americano revelou que a população feminina pode apresentar menores chances de se envolver com o uso de drogas, mas tende a ter um consumo em maior quantidade de crack do que as outras drogas, porém, os homens tendem a usar diferentes substâncias de forma simultânea (ANDRETTA I, et al., 2018; Weiss CV, et al., 2018; ABREU AMM, et al., 2018)

A idade dos usuários variou de 20 a 65 anos, com média de 40,1 anos. O uso abusivo de álcool e outras drogas têm sido considerados uns dos problemas de saúde pública mundial, pois afeta vários pontos da vida do usuário, provocando mudanças no âmbito social e familiar, com isso, as mortes acidentais e intencionais associadas ao uso dessas substâncias representam umas das principais causas evitáveis de morte entre a população de 15 a 24 anos de idade (TAVARES MLO, et al., 2017; REIS LM, OLIVEIRA MLF, 2017).

As pesquisas mostram que o álcool é a droga lícita mais utilizada, sendo citada por $90,9 \%$ dos pesquisados, seguido pelo uso do cigarro com $72,7 \%$. No Brasil, a dependência do álcool atinge cerca de $10 \%$ da população. O seu consumo tem se tornado excessivo ao longo de todos os tempos, podendo desenvolver diversos danos, sejam eles biológicos, psicológicos ou socioculturais (WEISS CV, et al., 2018; FERNANDES MA, et al., 2018; BAKARI I, MANN R, 2019).

Com relação às drogas ilícitas, a mais utilizada é o crack $(81,8 \%)$, geralmente estando associada ao consumo do álcool, sendo um hábito o consumo de múltiplas drogas entre os usuários, justificado como método para conter síndromes de abstinência provocada pela falta da droga. Em contraposição, pesquisas afirmam que a droga ilícita mais utilizada entre os usuários é a maconha, sendo a preferida entre os adolescentes (FERNANDES MA, et al., 2018; TAVARES MLO, et al., 2017).

O nível de escolaridade predominante nos usuários é o ensino fundamental incompleto (34,0\%), sendo em menor número aqueles que apresentam curso superior (2,7\%) e 55,0\% apresentavam entre um e dois salários mínimos, com isso, deduz-se que, quanto maior a renda e a escolaridade, menor o consumo dessas substâncias, estando relacionadas com a educação nas escolas, bem como a formação, ou seja, quanto maior a informação, melhor será a compreensão dessas pessoas no sentido da qualidade de vida e promoção a saúde (ABREU AMM, et al., 2016).

A renda dos usuários revela um aspecto importante, visto que essa situação pode agravar problemas relacionados à vulnerabilidade social, sendo um dos principais riscos relacionados ao uso de drogas, estando diretamente ligado a problemas secundários como a criminalidade, pois o dependente carece de dinheiro para a manutenção de consumo de substâncias (FERNANDES MA, et al., 2018).

Quanto à religião, $81,8 \%$ dos usuários afirmam ser católicos e o grupo menos prevalente são os evangélicos. Em contrapartida, Abreu AMM, et al. (2016), relatam que os usuários são em sua maioria evangélicos e apenas $47,5 \%$ católicos. Estudos mostram que a prática religiosa e o uso de álcool e drogas são dimensões que expressam os significados das experiências pessoais e sociais do indivíduo. Essas dimensões se refletem em impactos sobre a saúde física e mental, sobre comportamentos de risco e sobre o desenvolvimento psicossocial. Estudar as relações dessas dimensões é fundamental. Diferentes estudos associam não ter religião, ter pouca crença religiosa e não frequentar igreja e cultos ao maior uso de álcool e drogas (FELIPE AOB, et al., 2015; FERNANDES MA, et al., 2018). 
Em relação ao estado civil, a predominância é de solteiros sendo 75,9\%. Tanto na maternidade quanto na paternidade, a maior porcentagem é de apenas um filho. Já com relação a predominância da cor, 31,5\% se auto declara parda. A literatura aponta o fato de não viver com um parceiro fixo, não praticar alguma religião, insatisfação com a situação financeira e sofrer discriminação configuram-se como fatores de risco para o consumo problemático de droga (WEBSTER-CORRADI CM; DONATO-GHERARDI ECS, 2016). (REIS LM, OLIVEIRA MLF, 2017; FERNANDES MA, et al., 2018; TASSINARI TT, et al., 2018; WEISS CV, et al., 2018).

Além da curiosidade, o abandono de pais, filhos e companheiros, são umas das motivações iniciais que levam o usuário a entrar no mundo das drogas, como também, o convívio com os amigos e desilusões amorosas. Geralmente, o primeiro contato com as drogas ainda acontece na infância ou adolescência, comumente antes dos 18 anos, por influência da família ou pelo meio social das quais os dependentes estão inseridos (REIS LM, OLIVEIRA MLF, 2017; SIQUEIRA DF, et al., 2018; TASSINARI TT, et al., 2018; ELICKER E, et al., 2015).

\section{Importância do Serviço de Álcool e outras Drogas}

De acordo com Varela et al. (2016), o uso abusivo e a dependência de crack, álcool e diversas outras drogas, configura-se como um grave problema de saúde pública na sociedade moderna, tornando-se ponto central de várias políticas públicas no contexto brasileiro, requerendo a implantação de ações e serviços devidamente ordenado, bem articulados e com resolutividade frente a este problema, principalmente do Sistema Único de Saúde (SUS).

Com a instituição da Lei Federal de no 10.216/01, que autenticou o movimento da reforma psiquiátrica na área da saúde mental no Brasil, o interesse pela implementação de políticas sobre álcool e outras drogas no país passa a ganhar visibilidade, no intuito de priorizar a rede de cuidados extra-hospitalares, direcionando mais esforços para abranger a atenção integral a saúde ao perfil de pessoas que estão intimamente envolvidas com o consumo de drogas (MACHADO LV, BOARINI ML, 2013).

Atualmente, os Centros de Atenção Psicossocial - CAPS representam o centro de organização da assistência em saúde mental no Brasil. O Centro de Atenção Psicossocial Álcool e Drogas (CAPS AD) identifica e acolhe pessoas com problemas decorrentes do uso abusivo de drogas, com a intenção de proporcionar uma melhor assistência a esses indivíduos sendo de caráter comunitário e territorial, constituído por uma equipe multidisciplinar, tendo como principal objetivo promover a reabilitação de seus usuários (CARVALHO MRS, et al., 2017; SENA ELS, et al., 2017).

Objetiva-se com o CAPS, atender a população de acordo com a sua área de cobertura, por meio do acompanhamento clínico, promovendo a ressocialização dos usuários através do acesso ao lazer, trabalho, o fortalecimento dos laços com a família, com a comunidade e o exercício dos direitos civis Suas ações oferecem ao usuário a atenção no âmbito preventivo, na sua reabilitação, diante do fortalecimento de fatores de prevenção, proteção e tratamento tendo como meio as intervenções terapêuticas (TASSINARI TT, et al., 2018; SIQUEIRA DF, et al., 2018; TREVISAN ER, CASTRO SC, 2019).

Esse serviço surgiu como modelo substitutivo aos hospitais psiquiátricos pré-existentes e visam garantir aos usuários uma nova forma de cuidados com a saúde, possibilitando uma nova perspectiva da reinserção social, além da busca pela abstinência das substâncias psicoativas. O CAPS AD promove a saúde dos usuários e da população em geral, eliminando o foco da atenção centrada no controle e na repressão, por meio das atividades como participação em grupos ou oficinas para que ocorra o compartilhamento de vivências entre os participantes, contribuindo na identificação de pontos de apoio, de potencialidades e maneiras de trabalhar com as expectativas relacionadas ao uso e ao tratamento (BOSKA et al., 2018; SIQUEIRA DF, et al., 2018; TASSINARI TT, et al., 2018).

Pesquisas brasileiras referente a temática em questão vem apontando novos índices de que 0 estabelecimento dessa rede de cuidados é desafiante. Embora haja um déficit de estudos relacionados a temática que investiguem a articulação de diferentes serviços de saúde para atender o usuário de álcool, crack e outras drogas, existe alguns registros na literatura de casos que evidenciam sinais de fragilidades da atenção aos usuários que consequentemente afeta na efetivação deste processo e, por conseguinte, no atendimento e na resolutividade dos casos (MACHADO LV, BOARINI ML, 2013). 
No CAPS AD o cuidado deve ser pensado levando em conta a Redução de Danos (RD), estratégia que propõe o fortalecimento dos vínculos culturais e comunitários, o respeito à autonomia do sujeito e considera a rede de dispositivos de cuidados onde o sujeito está inserido um ponto primordial para a atenção integral, tendo em vista a superação da maneira reducionista de ver os sujeitos, muitas vezes, restringidos ao campo da marginalização e criminalidade (SENA ELS, et al., 2017).

Ao vivenciarem as perdas no âmbito familiar, os usuários buscam o tratamento no CAPS, com a intenção de resgatar esse vínculo afetivo. $\mathrm{O}$ acompanhamento familiar na vida do usuário é necessário, pois influencia diretamente num bom desenvolvimento na evolução do tratamento e na duração da abstinência, bem como, recuperar valores como a confiança, encontros prazerosos, além do respeito da família (SIQUEIRA DF, et al., 2018).

Sendo assim, surge a compreensão de que a atenção a esses usuários corresponde a uma atitude de responsabilização, de ocupação e de envolvimento com o outro, partindo do princípio de que o paciente é corresponsável por seu próprio cuidado, não um mero objeto de intervenção (SENA ELS, et al., 2017).

Os usuários dos CAPS AD percebem mudanças em suas vidas após o início do tratamento, sendo as mais significativas: maior confiança em si próprio, melhoria em seu humor, sentimento de maior interesse pela vida, capacidade de suportar situações difíceis, melhor qualidade do sono, e quando o usuário se encontra em tratamento, geralmente, há um controle maior sobre o seu consumo (BOSKA GA, et al., 2018).

\section{CONSIDERAÇÕES FINAIS}

O presente estudo permitiu compreender o perfil dos usuários de substâncias psicoativas, bem como a motivação inicial para o seu uso. Constatou-se que a maior parte desse público eram homens solteiros com apenas um filho, com a faixa etária entre 20 e 60 anos, a maioria não havia concluído o ensino fundamental, apresentando entre 1 e 2 salários mínimos, predominando a cor parda e o álcool como a droga lícita mais utilizada. No Brasil temos os Centros de Atenção Psicossocial - CAPS que representam o centro de organização da assistência em saúde mental que visam garantir aos usuários uma nova forma de cuidados com a saúde, possibilitando uma nova perspectiva da reinserção social, além da busca pela abstinência das substâncias psicoativas que promove a saúde dos usuários e da população em geral, eliminando o foco da atenção centrada no controle e na repressão, por meio das atividades como participação em grupos ou oficinas para que ocorra o compartilhamento de vivências entre os participantes, contribuindo na identificação de pontos de apoio, de potencialidades e maneiras de trabalhar com as expectativas relacionadas ao uso e ao tratamento.

\section{REFERÊNCIAS}

1. ABREU AMM, et al. Perfil do Consumo de Substâncias Psicoativas e sua Relação com as Características Sociodemográficas: uma Contribuição para Intervenção Breve na Atenção Primária à Saúde. Texto \& Contexto Enfermagem, 2018; 25(4): 1-12.

2. ANDRETTA I, et al. Sintomas de Depressão, Ansiedade e Estresse em Usuários de Drogas em Tratamento em Comunidades Terapêuticas. Psico-USF, Bragança Paulista, 2018; 23(2): 361-373.

3. BAKARI I, MANN R. Atitude em relação a pessoas que abusam drogas em uma comunidade urbana de woodbrook no porto de espanha - trinidad \& tobago. Texto \& Contexto Enfermagem, 2019; 28(18): 1-10.

4. BERALDO JB, et al. Rev. Enfermagem e Saúde Coletiva, 2018; 3(1): 24-32.

5. BOSKA GA, et al. Mudanças Percebidas por Usuários de Centros de Atenção Psicossocial em Álcool e Outras Drogas. Rev enferm UFPE, 2018; 12(2): 439-446.

6. CARVALHO MRS, et al. Motivações e repercussões do consumo de crack: o discurso coletivo de usuários de um Centro de Atenção Psicossocial. Escola Anna Nery. 2017; 21(3): 1-10.

7. CHAVES JJF, KHENTI A. Knowledge of the consequences and use of drugs for Costa Rica university students. Texto \& Contexto Enfermagem, 2019; 28(1): 416-425.

8. COSENTINO SF, et al. Características de Cuidadores Familiares e de Usuários de Drogas. Rev. enferm. UFPE, 2017; 11(6): 2400-2407.

9. ELICKER E, et al. Uso de álcool, tabaco e outras drogas por adolescentes escolares de Porto Velho-RO, Brasil. Epidemiol. Serv. Saúde v.24 n.3 Brasília set. 2015.

10. FELIPE AOB, et al. Espiritualidade e religião como protetores ao uso de drogas em adolescente. SMAD, Rev. Eletrônica Saúde Mental Álcool Drog. (Ed. port.), Ribeirão Preto, v. 11, n. 1, p. 49-58, mar. 2015.

11. FERNANDES MA, et al. Caracterização de Dependentes Químicos em Tratamento em uma Comunidade Terapêutica. REUOL- Revista de Enfermagem UFPE, 2018; 12(6): 1610-1616. 
12. LIMA DWC, et al. Práticas dos Enfermeiros às Pessoas em Crise Psíquica em Decorrência do Uso Abusivo de Drogas. Rev Enferm UFSM, 2018; 8(1): 18-32.

13. LUCCHESE R, et al. Transtorno Mental Comum entre Indivíduos que Abusam de Álcool e Drogas: Estudo Transversal. Texto \& Contexto Enfermagem, 2017; 26(1): 87-95.

14. MACHADO LV, BOARINI ML. Políticas sobre drogas no Brasil: a estratégia de redução de danos. Psicol. cienc. Prof, 2013; 33(3): 580-95.

15. NUNES JT, et al. Assistência de Enfermagem aos Usuários de Drogas Psicoativas em Sofrimento Psíquico: Relato de Experiência. Revista de Enfermagem da UFPI, 2017; 6(2): 74-78.

16. QUINDERÉ PH, et al. Rede de Atenção Psicossocial:qual o lugar da saúde mental? PhysisRevista de Saúde Coletiva, Rio de Janeiro, 24 [1]: 253-271, 2014

17. REIS LM, OLIVEIRA MLF. Vulnerabilidade social em famílias que convivem com comportamento aditivo por tempo prolongado. Acta Paul Enferm, 2017; 30(4):412-419.

18. SENA ELS, et al. Ambiguidade do Cuidado na Vivência do Consumidor de Drogas. Rev. Gaúcha Enferm, 2017; 38(2): 127-138.

19. SIQUEIRA DF, et al. Motivos Atribuídos por Usuários à Procura de Tratamento em um Centro de Atenção Psicossocial Álcool e Drogas. REME - Revista Mineira de Enfermagem, 2018; 22(1082): 1-7.

20. TASSINARI TT, et al. Caracterização de Mulheres em Tratamento Devido ao Uso de Drogas. Rev. enferm. UFPE, 2018; 12(12): 3344-3351.

21. TAVARES MLO, et al. Perfil de Adolescentes e Vulnerabilidade Para o Uso de Álcool e Outras Drogas. Rev. enferm. UFPE, 2017; 11(10): 3906-3912.

22. TREVISAN ER, CASTRO SC. Centros de Atenção Psicossocial - álcool e drogas: perfil dos usuários. Saúde debate 43 (121) 05 Ago 2019Apr-Jun 2019.

23. VARELA DSS, et al. Rede de saúde no atendimento ao usuário de álcool, crack e outras drogas. Esc Anna Nery, 2016; 20(2): 296-302.

24. WEBSTER-CORRADI CM, DONATO-GHERARDI ECS. Fatores associados ao consumo problemático de drogas entre pacientes psiquiátricos ambulatoriais. Rev. Latino-Am. Enfermagem. 24:e2815, 2016.

25. WEISS CV, et al. Prevalência da dependência de álcool em usuários de substâncias psicoativas. Rev Enferm UFPI, 2018; 7(1): 20-25. 